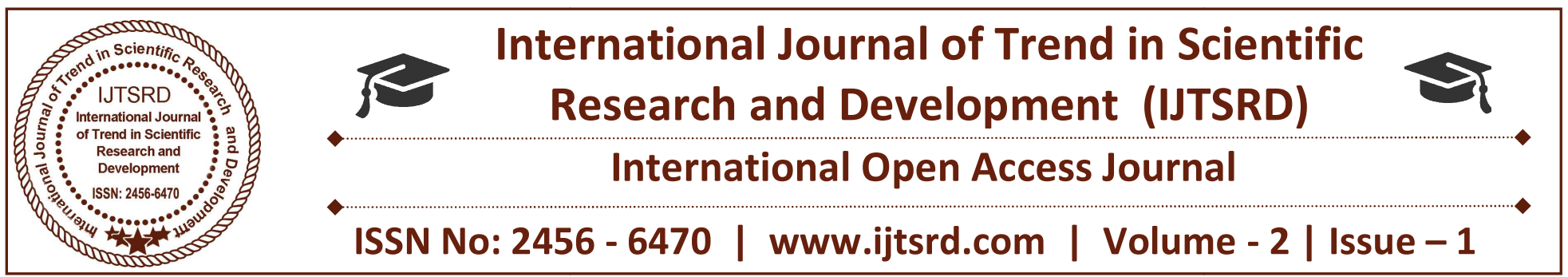

\title{
Energy data collection by survey and its comparison with bill data
}

Vikrram Singh Meena

Centre for Energy and Environment, Malaviya

National Institute of Technology, Jaipur, India

\author{
Dr. G. D. Agrawal \\ Centre for Energy and Environment, Malaviya \\ National Institute of Technology, Jaipur, India
}

\section{ABSTRACT}

In the Implementation of any electrical power generation project, the basic requirement is the total electrical energy demand estimation. In order to estimate the total electrical energy demand, various methods can be used but neither of those methods can give the exact demand. Therefore approximate analysis of energy demand is always taken in to the consideration. In this study three villages are selected in Rajasthan state and electrical energy demand of those villages is estimated. For the estimation of energy demands two sources of data are taken in to consideration. i.e. survey data and bill data. Survey data is collected by a quessionaire based survey and bill data are taken from electricity Distribution Company for the three selected village. On the basis of these two data, energy consumption parameters such as Energy consumption of village in $\mathrm{MWh} /$ annum, Energy consumption of family in $\mathrm{kWh} /$ annum, Per capita Energy consumption in $\mathrm{kWh}$ /annum, Energy consumption of village in $\mathrm{MWh} / \mathrm{month}$, Energy consumption of family in $\mathrm{kWh} /$ month, Per capita Energy consumption in $\mathrm{kWh} /$ month and Average Power in $\mathrm{kW} /$ month are calculated for each village .these energy consumption parameters are calculated in both the cases i.e. by using survey data and by using bill data, and comparison has made in tabular manner as well as graphical manner in order to observe deviation between the parameters properly.

\section{INTRODUCTION}

Jhar, Mansarkheri and Khijooriya jatan are the three selected villages for the study which are located in
Bassi sub division of Jaipur district in Rajasthan (latitude $26^{\circ} 83^{\prime} \mathbf{N}$, longitude $76^{\circ} 05^{\prime} \mathbf{E}$, altitude $351 \mathrm{~m}$ ).

Prasad et al. [1] showed an overview about the different facets of planning of energy with the help of various literatures. They showed the uncertainties, errors and risks which are involved in very well manner with the energy planning and continuously affecting the planning results. Urmee et al. [2] presented a study in which they showed the reasons of slow progress of renewable energy technologies based electrification. They suggested on the basis of literature reviewed on renewable energy based electrification that better coordination is very much required between the national level agencies and regional agencies which are actively involved in the development and the implementation of renewable energy based electrification program. Kautto and Peck [3] examined the potential of Biomass planning at regional level to support the Biomass planning at national level, European Union target achievement and addressing the concerns which are involved with increased bio energy production. Singh [4] presented a study which investigated the commercial availability of the biomass from the agricultural activities in Punjab in order to generate power from distributed power generation facilities. He estimated the total production of crop generated residues which is 55.396 Mt. Out of this total crop residue around $40.17 \%$ which is $22.315 \mathrm{Mt}$ is found to be surplus which can be used as biomass in power plants for the generation of electricity 
Bhandari and Jana [5] presented the impacts of characteristics of individual rural households on the priority of electrical energy from solar energy based system. This study is based upon the rural energy survey which is conducted in a coastal village of Indian sundarban. Ishan and Pallav [6] evaluated the solar power generation based on concentrating technology (CSP), technically as well as financially in India. They concluded that CSP based power generation facilities are financial feasible for selected part of India like north-western part especially Rajasthan and Gujarat states.

\section{DATA COLLECTION AND COMPARISION}

Energy consumption data of the villages Jhar, Mansarkheri and Khi.Jatan is collected by questionnaire based survey. In each village 50 households are selected in equal proportion of lower income group, moderate income group and higher income group in order to maintain reliability of the study and for better analysis and data is collected. The population related data of villages is also collected from Census Authority of the Rajasthan in which the population of the three villages is mentioned and tabulated in table-1.

\section{Table-1: Population data}

\begin{tabular}{|c|c|c|c|}
\hline Particulars/Village & Jhar & Mansarkheri & $\begin{array}{c}\text { Khi. } \\
\text { Jatan }\end{array}$ \\
\hline Households & 757 & 513 & 198 \\
\hline Population & 4683 & 3662 & 943 \\
\hline
\end{tabular}

Table-2: Energy data collected by Survey

\begin{tabular}{|c|c|c|c|c|c|c|c|c|c|}
\hline Village & \multicolumn{3}{|c|}{ Jhar } & \multicolumn{3}{c|}{ Mansarkheri } & \multicolumn{3}{c|}{ Khi. Jatan } \\
$\begin{array}{c}\text { Household } \\
\text { No. }\end{array}$ & $\begin{array}{c}\text { Bi-monthly } \\
\text { Avg }\end{array}$ & $\begin{array}{c}\text { Monthly } \\
\text { Avg }\end{array}$ & Annual & $\begin{array}{c}\text { Bi-monthly } \\
\text { Avg }\end{array}$ & $\begin{array}{c}\text { Monthly } \\
\text { Avg }\end{array}$ & Annual & $\begin{array}{c}\text { Bi-monthly } \\
\text { Avg }\end{array}$ & $\begin{array}{c}\text { Monthly } \\
\text { Avg }\end{array}$ & Annual \\
\hline $\mathbf{1}$ & 100 & 50 & 600 & 100 & 50 & 600 & 100 & 50 & 600 \\
$\mathbf{2}$ & 50 & 25 & 300 & 300 & 150 & 1800 & 100 & 50 & 600 \\
\hline $\mathbf{3}$ & 100 & 50 & 600 & 100 & 50 & 600 & 200 & 100 & 1200 \\
\hline $\mathbf{4}$ & 100 & 50 & 600 & 500 & 250 & 3000 & 100 & 50 & 600 \\
$\mathbf{5}$ & 200 & 100 & 1200 & 200 & 100 & 1200 & 100 & 50 & 600 \\
\hline $\mathbf{6}$ & 30 & 15 & 180 & 200 & 100 & 1200 & 400 & 200 & 2400 \\
\hline $\mathbf{7}$ & 100 & 50 & 600 & 100 & 50 & 600 & 100 & 50 & 600 \\
\hline $\mathbf{8}$ & 100 & 50 & 600 & 300 & 150 & 1800 & 150 & 75 & 900 \\
\hline $\mathbf{9}$ & 300 & 150 & 1800 & 150 & 75 & 900 & 100 & 50 & 600 \\
\hline $\mathbf{1 0}$ & 100 & 50 & 600 & 200 & 100 & 1200 & 100 & 50 & 600 \\
\hline $\mathbf{1 1}$ & 100 & 50 & 600 & 500 & 250 & 3000 & 100 & 50 & 600 \\
\hline $\mathbf{1 2}$ & 50 & 25 & 300 & 100 & 50 & 600 & 100 & 50 & 600 \\
\hline $\mathbf{1 3}$ & 200 & 100 & 1200 & 200 & 100 & 1200 & 100 & 50 & 600 \\
\hline $\mathbf{1 4}$ & 200 & 100 & 1200 & 100 & 50 & 600 & 300 & 150 & 1800 \\
\hline $\mathbf{1 5}$ & 100 & 50 & 600 & 500 & 250 & 3000 & 50 & 25 & 300 \\
\hline $\mathbf{1 6}$ & 50 & 25 & 300 & 200 & 100 & 1200 & 100 & 50 & 600 \\
\hline $\mathbf{1 7}$ & 100 & 50 & 600 & 150 & 75 & 900 & 100 & 50 & 600 \\
\hline $\mathbf{1 8}$ & 300 & 150 & 1800 & 500 & 250 & 3000 & 100 & 50 & 600 \\
\hline $\mathbf{1 9}$ & 150 & 75 & 900 & 100 & 50 & 600 & 100 & 50 & 600 \\
\hline $\mathbf{2 0}$ & 200 & 100 & 1200 & 200 & 100 & 1200 & 100 & 50 & 600 \\
\hline $\mathbf{2 1}$ & 100 & 50 & 600 & 500 & 250 & 3000 & 50 & 25 & 300 \\
\hline $\mathbf{2 2}$ & 100 & 50 & 600 & 50 & 25 & 300 & 100 & 50 & 600 \\
\hline $\mathbf{2 3}$ & 250 & 125 & 1500 & 50 & 25 & 300 & 100 & 50 & 600 \\
\hline $\mathbf{2 4}$ & 100 & 50 & 600 & 100 & 50 & 600 & 100 & 50 & 600 \\
\hline $\mathbf{2 5}$ & 100 & 50 & 600 & 100 & 50 & 600 & 200 & 100 & 1200 \\
\hline $\mathbf{2 6}$ & 200 & 100 & 1200 & 100 & 50 & 600 & 100 & 50 & 600 \\
\hline $\mathbf{2 7}$ & 500 & 250 & 3000 & 100 & 50 & 600 & 150 & 75 & 900 \\
\hline $\mathbf{2 8}$ & 300 & 150 & 1800 & 50 & 25 & 300 & 150 & 75 & 900 \\
\hline & & & & & & & & & \\
\hline
\end{tabular}


International Journal of Trend in Scientific Research and Development (IJTSRD) ISSN: 2456-6470

\begin{tabular}{|c|c|c|c|c|c|c|c|c|c|}
\hline 29 & 50 & 25 & 300 & 100 & 50 & 600 & 100 & 50 & 600 \\
\hline 30 & 100 & 50 & 600 & 100 & 50 & 600 & 150 & 75 & 900 \\
\hline 31 & 50 & 25 & 300 & 100 & 50 & 600 & 100 & 50 & 600 \\
\hline 32 & 50 & 25 & 300 & 50 & 25 & 300 & 150 & 75 & 900 \\
\hline 33 & 150 & 75 & 900 & 50 & 25 & 300 & 300 & 150 & 1800 \\
\hline 34 & 100 & 50 & 600 & 300 & 150 & 1800 & 100 & 50 & 600 \\
\hline 35 & 150 & 75 & 900 & 150 & 75 & 900 & 400 & 200 & 2400 \\
\hline 36 & 150 & 75 & 900 & 50 & 25 & 300 & 300 & 150 & 1800 \\
\hline 37 & 350 & 175 & 2100 & 150 & 75 & 900 & 200 & 100 & 1200 \\
\hline 38 & 400 & 200 & 2400 & 100 & 50 & 600 & 100 & 50 & 600 \\
\hline 39 & 500 & 250 & 3000 & 400 & 200 & 2400 & 50 & 25 & 300 \\
\hline 40 & 350 & 175 & 2100 & 50 & 25 & 300 & 100 & 50 & 600 \\
\hline 41 & 250 & 125 & 1500 & 150 & 75 & 900 & 100 & 50 & 600 \\
\hline 42 & 500 & 250 & 3000 & 1000 & 500 & 6000 & 50 & 25 & 300 \\
\hline 43 & 100 & 50 & 600 & 100 & 50 & 600 & 100 & 50 & 600 \\
\hline 44 & 300 & 150 & 1800 & 100 & 50 & 600 & 100 & 50 & 600 \\
\hline 45 & 150 & 75 & 900 & 50 & 25 & 300 & 100 & 50 & 600 \\
\hline 46 & 200 & 100 & 1200 & 50 & 25 & 300 & 100 & 50 & 600 \\
\hline 47 & 100 & 50 & 600 & 50 & 25 & 300 & 100 & 50 & 600 \\
\hline 48 & 200 & 100 & 1200 & 50 & 25 & 300 & 100 & 50 & 600 \\
\hline 49 & 50 & 25 & 300 & 100 & 50 & 600 & 100 & 50 & 600 \\
\hline 50 & 100 & 50 & 600 & 50 & 25 & 300 & 100 & 50 & 600 \\
\hline $\mathbf{P}$ & 8630 & 4315 & 51780 & 9050 & 4525 & 54300 & 6550 & 3275 & 39300 \\
\hline $\mathbf{Q}$ & 172.6 & 86.3 & 1035.6 & 181 & 90.5 & 1086 & 131 & 65.5 & 786 \\
\hline $\mathbf{R}$ & 130.66 & 65.33 & 783.95 & 92.85 & 46.43 & 557.12 & 25.94 & 12.97 & 155.63 \\
\hline $\mathbf{S}$ & 27.90 & 13.95 & 167.40 & 25.36 & 12.68 & 152.13 & 27.51 & 13.75 & 165.03 \\
\hline
\end{tabular}

P-Consumptions of 50 households in kWh, Q-kWh per family, R-Total consumption of village in MWh, S-Per capita consumption in $\mathrm{kWh}$

Table-3: Bill data for village Jhar

\begin{tabular}{|c|c|c|c|c|c|c|c|c|}
\hline Household No. & Jan-Fel & Mar-Apr & May-Jun & Jul-Aug & Sep-Oct & Nov-Dec & Annual & Monthly Avg \\
\hline $\mathbf{1}$ & 96 & 95 & 98 & 17 & 100 & 100 & 506 & 42.17 \\
\hline $\mathbf{2}$ & 43 & 28 & 74 & 32 & 40 & 52 & 269 & 22.42 \\
\hline $\mathbf{3}$ & 40 & 30 & 79 & 100 & 100 & 100 & 449 & 37.42 \\
\hline $\mathbf{4}$ & 80 & 89 & 122 & 105 & 215 & 401 & 1012 & 84.33 \\
\hline $\mathbf{5}$ & 237 & 104 & 393 & 92 & 192 & 279 & 1297 & 108.08 \\
\hline $\mathbf{6}$ & 6 & 12 & 10 & 30 & 22 & 17 & 97 & 8.08 \\
\hline $\mathbf{7}$ & 100 & 100 & 100 & 100 & 100 & 100 & 600 & 50.00 \\
\hline $\mathbf{8}$ & 100 & 100 & 100 & 100 & 100 & 100 & 600 & 50.00 \\
\hline $\mathbf{9}$ & 276 & 189 & 440 & 333 & 248 & 549 & 2035 & 169.58 \\
\hline $\mathbf{1 0}$ & 30 & 82 & 55 & 45 & 67 & 826 & 1105 & 92.08 \\
\hline $\mathbf{1 1}$ & 61 & 48 & 142 & 173 & 84 & 237 & 745 & 62.08 \\
\hline $\mathbf{1 2}$ & 0 & 0 & 0 & 100 & 100 & 1 & 201 & 16.75 \\
\hline $\mathbf{1 3}$ & 241 & 61 & 227 & 423 & 198 & 274 & 1424 & 118.67 \\
\hline $\mathbf{1 5}$ & 209 & 512 & 223 & 48 & 233 & 408 & 1633 & 136.08 \\
\hline $\mathbf{1 6}$ & 100 & 100 & 100 & 100 & 100 & 100 & 600 & 50.00 \\
\hline $\mathbf{1 7}$ & 100 & 51 & 32 & 8 & 33 & 40 & 264 & 22.00 \\
\hline
\end{tabular}


International Journal of Trend in Scientific Research and Development (IJTSRD) ISSN: 2456-6470

\begin{tabular}{|c|c|c|c|c|c|c|c|c|}
\hline 18 & 100 & 134 & 100 & 400 & 947 & 350 & 2031 & 169.25 \\
\hline 19 & 97 & 33 & 40 & 186 & 186 & 216 & 758 & 63.17 \\
\hline 20 & 42 & 15 & 117 & 198 & 617 & 54 & 1043 & 86.92 \\
\hline 21 & 117 & 100 & 117 & 117 & 117 & 117 & 685 & 57.08 \\
\hline 22 & 9 & 75 & 100 & 100 & 100 & 100 & 484 & 40.33 \\
\hline 23 & 193 & 132 & 524 & 211 & 412 & 406 & 1878 & 156.50 \\
\hline 24 & 31 & 150 & 108 & 9 & 208 & 84 & 590 & 49.17 \\
\hline 25 & 109 & 149 & 164 & 41 & 123 & 153 & 739 & 61.58 \\
\hline 26 & 88 & 52 & 324 & 303 & 206 & 141 & 1114 & 92.83 \\
\hline 27 & 254 & 208 & 1170 & 873 & 1124 & 713 & 4342 & 361.83 \\
\hline 28 & 228 & 32 & 434 & 233 & 150 & 1075 & 2152 & 179.33 \\
\hline 29 & 32 & 100 & 84 & 27 & 52 & 34 & 329 & 27.42 \\
\hline 30 & 100 & 100 & 100 & 100 & 100 & 100 & 600 & 50.00 \\
\hline 31 & 5 & 30 & 39 & 3 & 35 & 98 & 210 & 17.50 \\
\hline 32 & 36 & 75 & 46 & 33 & 134 & 143 & 467 & 38.92 \\
\hline 33 & 137 & 150 & 150 & 150 & 150 & 150 & 887 & 73.92 \\
\hline 34 & 0 & 200 & 100 & 115 & 99 & 107 & 621 & 51.75 \\
\hline 35 & 100 & 49 & 152 & 299 & 225 & 87 & 912 & 76.00 \\
\hline 36 & 114 & 167 & 129 & 127 & 150 & 150 & 837 & 69.75 \\
\hline 37 & 253 & 120 & 673 & 242 & 597 & 756 & 2641 & 220.08 \\
\hline 38 & 126 & 260 & 912 & 586 & 391 & 391 & 2666 & 222.17 \\
\hline 39 & 79 & 121 & 101 & 111 & 100 & 2400 & 2912 & 242.67 \\
\hline 40 & 174 & 87 & 704 & 470 & 219 & 462 & 2116 & 176.33 \\
\hline 41 & 105 & 105 & 375 & 387 & 339 & 257 & 1568 & 130.67 \\
\hline 42 & 403 & 364 & 651 & 339 & 1065 & 411 & 3233 & 269.42 \\
\hline 43 & 82 & 88 & 150 & 47 & 171 & 210 & 748 & 62.33 \\
\hline 44 & 219 & 87 & 342 & 80 & 394 & 237 & 1359 & 113.25 \\
\hline 45 & 100 & 100 & 100 & 255 & 146 & 108 & 809 & 67.42 \\
\hline 46 & 343 & 447 & 756 & 97 & 79 & 117 & 1839 & 153.25 \\
\hline 47 & 100 & 100 & 100 & 75 & 100 & 100 & 575 & 47.92 \\
\hline 48 & 63 & 80 & 69 & 82 & 100 & 1050 & 1444 & 120.33 \\
\hline 49 & 68 & 18 & 51 & 45 & 62 & 37 & 281 & 23.42 \\
\hline 50 & 44 & 52 & 38 & 100 & 100 & 390 & 724 & 60.33 \\
\hline $\mathbf{P}$ & 5779 & 5682 & 11266 & 8345 & 11002 & 14938 & 57012 & 4751 \\
\hline $\mathbf{Q}$ & 115.58 & 113.64 & 225.32 & 166.9 & 220.04 & 298.76 & 1140.2 & 95.02 \\
\hline $\mathbf{R}$ & 87.49 & 86.03 & 170.57 & 126.34 & 166.57 & 226.16 & 863.16 & 71.93 \\
\hline $\mathbf{S}$ & 18.68 & 18.37 & 36.42 & 26.98 & 35.57 & 48.29 & 184.32 & 15.36 \\
\hline
\end{tabular}


International Journal of Trend in Scientific Research and Development (IJTSRD) ISSN: 2456-6470

Table-4: Bill data for village Mansarkheri

\begin{tabular}{|c|c|c|c|c|c|c|c|c|}
\hline Household No. & Jan-Feb & Mar-Apr & May-Jun & Jul-Aug & Sep-Oct & Nov-Dec & Annual & Monthly Avg \\
\hline 1 & 99 & 99 & 90 & 99 & 67 & 100 & 554 & 46.17 \\
\hline 2 & 192 & 131 & 58 & 504 & 275 & 777 & 1937 & 161.42 \\
\hline 3 & 69 & 149 & 39 & 142 & 102 & 19 & 520 & 43.33 \\
\hline 4 & 280 & 282 & 202 & 654 & 706 & 979 & 3103 & 258.58 \\
\hline 5 & 106 & 123 & 152 & 354 & 116 & 488 & 1339 & 111.58 \\
\hline 6 & 411 & 214 & 272 & 80 & 414 & 336 & 1727 & 143.92 \\
\hline 7 & 145 & 79 & 73 & 274 & 125 & 124 & 820 & 68.33 \\
\hline 8 & 243 & 152 & 71 & 612 & 490 & 466 & 2034 & 169.50 \\
\hline 9 & 114 & 92 & 99 & 176 & 116 & 206 & 803 & 66.92 \\
\hline 10 & 204 & 183 & 90 & 209 & 422 & 274 & 1382 & 115.17 \\
\hline 11 & 185 & 596 & 390 & 625 & 900 & 878 & 3574 & 297.83 \\
\hline 12 & 33 & 10 & 11 & 73 & 97 & 138 & 362 & 30.17 \\
\hline 13 & 98 & 459 & 35 & 295 & 139 & 226 & 1252 & 104.33 \\
\hline 14 & 172 & 31 & 40 & 150 & 150 & 233 & 776 & 64.67 \\
\hline 15 & 1494 & 187 & 70 & 683 & 608 & 316 & 3358 & 279.83 \\
\hline 16 & 128 & 133 & 158 & 510 & 94 & 302 & 1325 & 110.42 \\
\hline 17 & 302 & 240 & 255 & 0 & 300 & 150 & 1247 & 103.92 \\
\hline 18 & 583 & 312 & 257 & 508 & 754 & 588 & 3002 & 250.17 \\
\hline 19 & 100 & 100 & 100 & 135 & 66 & 206 & 707 & 58.92 \\
\hline 20 & 251 & 178 & 154 & 222 & 105 & 300 & 1210 & 100.83 \\
\hline 21 & 395 & 121 & 282 & 887 & 409 & 930 & 3024 & 252.00 \\
\hline 22 & 92 & 47 & 71 & 62 & 88 & 52 & 412 & 34.33 \\
\hline 23 & 52 & 35 & 30 & 100 & 68 & 82 & 367 & 30.58 \\
\hline 24 & 96 & 65 & 15 & 119 & 83 & 129 & 507 & 42.25 \\
\hline 25 & 87 & 66 & 74 & 100 & 96 & 56 & 479 & 39.92 \\
\hline 26 & 152 & 19 & 18 & 94 & 100 & 175 & 558 & 46.50 \\
\hline 27 & 63 & 23 & 37 & 191 & 227 & 166 & 707 & 58.92 \\
\hline 28 & 91 & 100 & 35 & 36 & 2 & 49 & 313 & 26.08 \\
\hline 29 & 27 & 118 & 15 & 119 & 46 & 68 & 393 & 32.75 \\
\hline 30 & 65 & 47 & 46 & 266 & 104 & 147 & 675 & 56.25 \\
\hline 31 & 150 & 150 & 100 & 127 & 8 & 150 & 685 & 57.08 \\
\hline 32 & 42 & 21 & 7 & 16 & 10 & 10 & 106 & 8.83 \\
\hline 33 & 28 & 37 & 20 & 18 & 9 & 22 & 134 & 11.17 \\
\hline 34 & 379 & 186 & 54 & 578 & 242 & 583 & 2022 & 168.50 \\
\hline 35 & 80 & 71 & 44 & 292 & 91 & 270 & 848 & 70.67 \\
\hline 36 & 5 & 13 & 6 & 62 & 18 & 7 & 111 & 9.25 \\
\hline 37 & 180 & 179 & 122 & 99 & 121 & 167 & 868 & 72.33 \\
\hline 38 & 15 & 100 & 100 & 100 & 100 & 100 & 515 & 42.92 \\
\hline 39 & 380 & 888 & 387 & 820 & 238 & 134 & 2847 & 237.25 \\
\hline 40 & 74 & 81 & 27 & 47 & 6 & 39 & 274 & 22.83 \\
\hline 41 & 156 & 115 & 58 & 206 & 145 & 204 & 884 & 73.67 \\
\hline 42 & 85 & 3630 & 1858 & 90 & 15 & 49 & 5727 & 477.25 \\
\hline 43 & 95 & 68 & 53 & 100 & 62 & 332 & 710 & 59.17 \\
\hline 44 & 82 & 60 & 83 & 91 & 8 & 201 & 525 & 43.75 \\
\hline 45 & 23 & 39 & 64 & 138 & 59 & 82 & 405 & 33.75 \\
\hline 46 & 95 & 1 & 69 & 213 & 29 & 121 & 528 & 44.00 \\
\hline 47 & 42 & 10 & 26 & 23 & 72 & 21 & 194 & 16.17 \\
\hline
\end{tabular}


International Journal of Trend in Scientific Research and Development (IJTSRD) ISSN: 2456-6470

\begin{tabular}{|c|c|c|c|c|c|c|c|c|}
\hline $\mathbf{4 8}$ & 99 & 72 & 29 & 90 & 33 & 13 & 336 & 28.00 \\
\hline $\mathbf{4 9}$ & 16 & 58 & 55 & 100 & 100 & 100 & 429 & 35.75 \\
\hline $\mathbf{5 0}$ & 36 & 150 & 7 & 65 & 43 & 63 & 364 & 30.33 \\
\hline $\mathbf{P}$ & 8391 & 10320 & 6408 & 11554 & 8678 & 11628 & 56979 & 4748 \\
\hline $\mathbf{Q}$ & 167.82 & 206.4 & 128.16 & 231.08 & 173.56 & 232.56 & 1139.6 & 94.97 \\
\hline $\mathbf{R}$ & 86.09 & 105.88 & 65.75 & 118.54 & 89.04 & 119.30 & $\mathbf{5 8 4 . 6 0}$ & 48.72 \\
\hline S & 23.51 & 28.91 & 17.95 & 32.37 & 24.31 & 32.58 & 159.64 & 13.30 \\
\hline
\end{tabular}

Table-5: Bill data for village Khijooriya jatan

\begin{tabular}{|c|c|c|c|c|c|c|c|c|}
\hline Household No. & Jan-Feb & Mar-Apr & May-Jun & Jul-Aug & Sep-Oct & Nov-Dec & Annual & Monthly Avg \\
\hline 1 & 152 & 66 & 59 & 93 & 172 & 126 & 668 & 55.67 \\
\hline 2 & 295 & 65 & 48 & 63 & 65 & 82 & 618 & 51.50 \\
\hline 3 & 100 & 100 & 100 & 178 & 1012 & 236 & 1726 & 143.83 \\
\hline 4 & 100 & 100 & 100 & 100 & 100 & 100 & 600 & 50.00 \\
\hline 5 & 100 & 225 & 26 & 55 & 381 & 218 & 1005 & 83.75 \\
\hline 6 & 887 & 38 & 1009 & 487 & 537 & 423 & 3381 & 281.75 \\
\hline 7 & 1 & 278 & 322 & 87 & 130 & 108 & 926 & 77.17 \\
\hline 8 & 95 & 43 & 305 & 174 & 134 & 52 & 803 & 66.92 \\
\hline 9 & 1 & 9 & 100 & 100 & 100 & 100 & 410 & 34.17 \\
\hline 10 & 100 & 100 & 100 & 100 & 100 & 100 & 600 & 50.00 \\
\hline 11 & 100 & 100 & 100 & 100 & 177 & 138 & 715 & 59.58 \\
\hline 12 & 100 & 100 & 100 & 50 & 100 & 79 & 529 & 44.08 \\
\hline 13 & 146 & 150 & 36 & 150 & 98 & 80 & 660 & 55.00 \\
\hline 14 & 888 & 74 & 436 & 171 & 213 & 257 & 2039 & 169.92 \\
\hline 15 & 100 & 100 & 250 & 1 & 1 & 1 & 453 & 37.75 \\
\hline 16 & 150 & 100 & 100 & 100 & 100 & 100 & 650 & 54.17 \\
\hline 17 & 1 & 33 & 100 & 100 & 100 & 100 & 434 & 36.17 \\
\hline 18 & 100 & 100 & 100 & 100 & 100 & 100 & 600 & 50.00 \\
\hline 19 & 100 & 100 & 100 & 100 & 100 & 100 & 600 & 50.00 \\
\hline 20 & 150 & 84 & 117 & 111 & 111 & 111 & 684 & 57.00 \\
\hline 21 & 100 & 94 & 100 & 9 & 47 & 64 & 414 & 34.50 \\
\hline 22 & 100 & 100 & 100 & 100 & 100 & 100 & 600 & 50.00 \\
\hline 23 & 100 & 100 & 2 & 100 & 100 & 100 & 502 & 41.83 \\
\hline 24 & 100 & 100 & 100 & 100 & 100 & 100 & 600 & 50.00 \\
\hline 25 & 478 & 62 & 391 & 77 & 80 & 83 & 1171 & 97.58 \\
\hline 26 & 100 & 100 & 0 & 242 & 59 & 52 & 553 & 46.08 \\
\hline 27 & 100 & 100 & 100 & 100 & 453 & 127 & 980 & 81.67 \\
\hline 28 & 61 & 48 & 100 & 230 & 172 & 172 & 783 & 65.25 \\
\hline 29 & 60 & 43 & 150 & 279 & 86 & 84 & 702 & 58.50 \\
\hline 30 & 100 & 100 & 444 & 162 & 48 & 78 & 932 & 77.67 \\
\hline 31 & 100 & 100 & 100 & 100 & 100 & 100 & 600 & 50.00 \\
\hline 32 & 150 & 153 & 152 & 124 & 124 & 124 & 827 & 68.92 \\
\hline 33 & 100 & 100 & 1404 & 79 & 45 & 74 & 1802 & 150.17 \\
\hline 34 & 100 & 100 & 100 & 45 & 68 & 50 & 463 & 38.58 \\
\hline 35 & 1034 & 91 & 770 & 415 & 98 & 58 & 2466 & 205.50 \\
\hline 36 & 1146 & 69 & 289 & 179 & 150 & 150 & 1983 & 165.25 \\
\hline 37 & 722 & 83 & 402 & 0 & 88 & 103 & 1398 & 116.50 \\
\hline 38 & 100 & 150 & 90 & 32 & 76 & 27 & 475 & 39.58 \\
\hline
\end{tabular}


International Journal of Trend in Scientific Research and Development (IJTSRD) ISSN: 2456-6470

\begin{tabular}{|c|c|c|c|c|c|c|c|c|}
\hline $\mathbf{3 9}$ & 1 & 21 & 41 & 26 & 64 & 47 & 200 & 16.67 \\
\hline $\mathbf{4 0}$ & 131 & 50 & 223 & 69 & 54 & 86 & 613 & 51.08 \\
\hline $\mathbf{4 1}$ & 100 & 100 & 100 & 100 & 100 & 143 & 643 & 53.58 \\
\hline $\mathbf{4 2}$ & 100 & 0 & 0 & 0 & 0 & 100 & 200 & 16.67 \\
\hline $\mathbf{4 3}$ & 46 & 42 & 63 & 100 & 100 & 100 & 451 & 37.58 \\
\hline $\mathbf{4 4}$ & 100 & 100 & 0 & 100 & 100 & 100 & 500 & 41.67 \\
\hline $\mathbf{4 5}$ & 100 & 150 & 150 & 150 & 122 & 1 & 673 & 56.08 \\
\hline $\mathbf{4 6}$ & 60 & 84 & 87 & 150 & 110 & 30 & 521 & 43.42 \\
\hline $\mathbf{4 7}$ & 95 & 59 & 78 & 92 & 128 & 62 & 514 & 42.83 \\
\hline $\mathbf{4 8}$ & 100 & 100 & 91 & 33 & 44 & 139 & 507 & 42.25 \\
\hline $\mathbf{4 9}$ & 100 & 100 & 100 & 100 & 100 & 100 & 600 & 50.00 \\
\hline $\mathbf{5 0}$ & 100 & 150 & 39 & 26 & 66 & 16 & 397 & 33.08 \\
\hline $\mathbf{P}$ & 9450 & 4614 & 9374 & 5739 & 6813 & 5181 & 41171 & 3431 \\
\hline $\mathbf{Q}$ & 189 & 92.28 & 187.48 & 114.78 & 136.26 & 103.62 & 823.42 & 68.62 \\
\hline $\mathbf{R}$ & 37.42 & 18.27 & 37.12 & 22.73 & 26.98 & 20.52 & $\mathbf{1 6 3 . 0 4}$ & 13.59 \\
\hline $\mathbf{S}$ & 39.68 & 19.38 & 39.36 & 24.10 & 28.61 & 21.76 & 172.89 & 14.41 \\
\hline
\end{tabular}

The data collected by the survey is the primary data of energy consumption of villages and approximate also therefore energy consumption data is also collected from electricity bills provided by the local electricity distribution company. The data collected by survey is tabulated in Table-2 and bill data are tabulated in table-3 to table-5. Sample calculation for Jhar Village is as follows-

Annual electricity consumption of 50 households $=$ $51780 \mathrm{kWh}$. Therefore annual electricity consumption per family $=\frac{51780}{50}=\mathbf{1 0 3 5 . 6 0} \mathbf{~ k W h}$
Total number of families $=757$

Therefore annual electricity consumption of whole village $=1035.60 \times 757=783949.20 \mathrm{kWh}=783.95$ $\mathrm{MWh}$

Total population $=4683$

Therefore annual per capita electricity consumption $=$ $\frac{783.95}{4683}=167.40 \mathrm{kWh}$

Some deviation is observed between collected data by the survey and collected data from electricity bills. To observe that deviation the consolidated data of electricity consumption both by electricity bills and survey are arranged in tabulated format in Table-6.

Table-6: Consolidated electricity consumption data of villages

\begin{tabular}{|c|c|c|c|c|c|c|}
\hline Particulars & X Bill & X survey & Y Bill & Y Survey & Z Bill & Z Survey \\
\hline A & 863.16 & 783.95 & 584.60 & 557.12 & 163.04 & 155.63 \\
\hline B & 1140.20 & 1035.60 & 1139.58 & 1086 & 823.42 & 786 \\
\hline C & 184.32 & 167.40 & 159.64 & 152.13 & 172.89 & 165.03 \\
\hline D & 71.93 & 65.33 & 48.72 & 46.43 & 13.59 & 12.97 \\
\hline E & 95.02 & 86.30 & 94.97 & 90.50 & 68.62 & 65.50 \\
\hline F & 15.36 & 13.95 & 13.30 & 12.68 & 14.41 & 13.75 \\
\hline G & 337.83 & 306.83 & 228.81 & 218.06 & 63.81 & 60.91 \\
\hline
\end{tabular}

A-Energy consumption of villages in $\mathrm{MWh} /$ annum, B-Energy consumption of family in $\mathrm{kWh} /$ annum, CPer capita Energy consumption in $\mathrm{kWh} /$ annum, DEnergy consumption of villages in $\mathrm{MWh} / \mathrm{month}$, E-
Energy consumption of family in $\mathrm{kWh} /$ month, F- Per capita Energy consumption in $\mathrm{kWh} /$ month, GAverage Power in $\mathrm{kW} /$ month, X- Jhar, Y-Mansarkheri and Z-Khijooriya jatan 


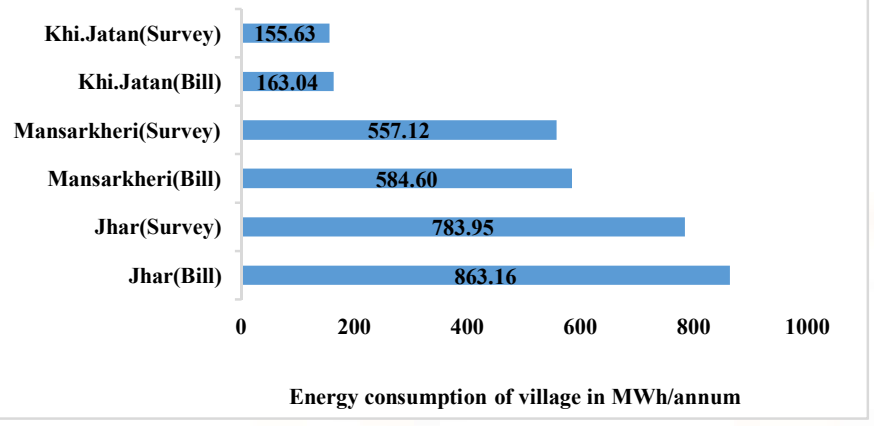

Figure -1: Energy consumption of village in MWh/annum

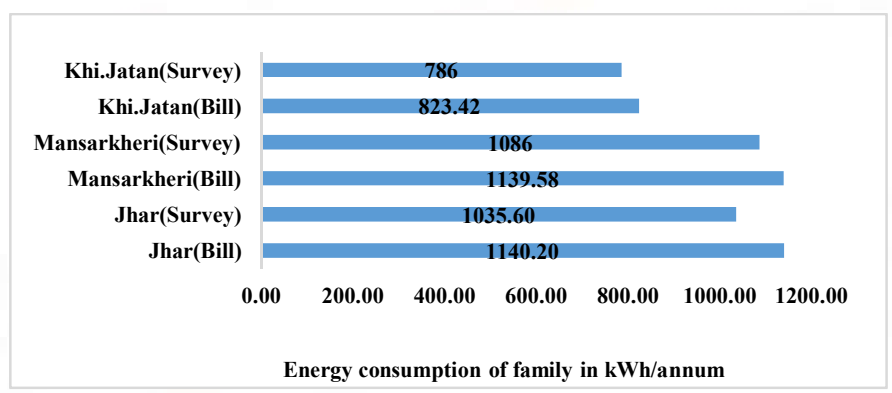

Figure-2: Energy consumption of family in $\mathrm{kWh} /$ annum

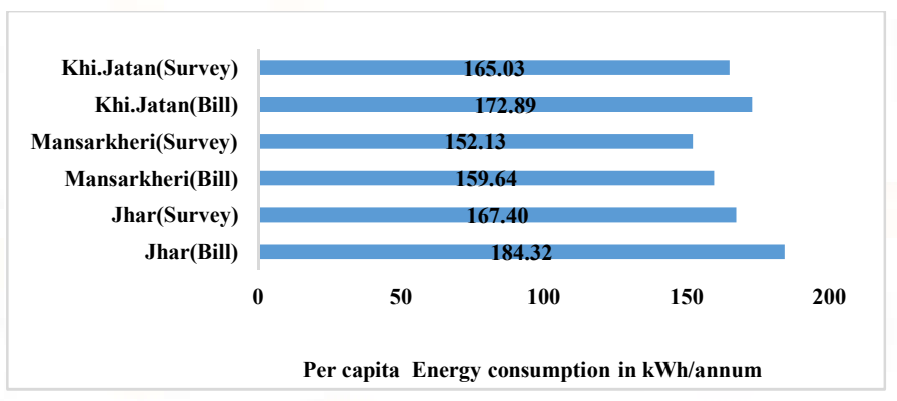

Figure-3: Per capita energy consumption in kWh/annum

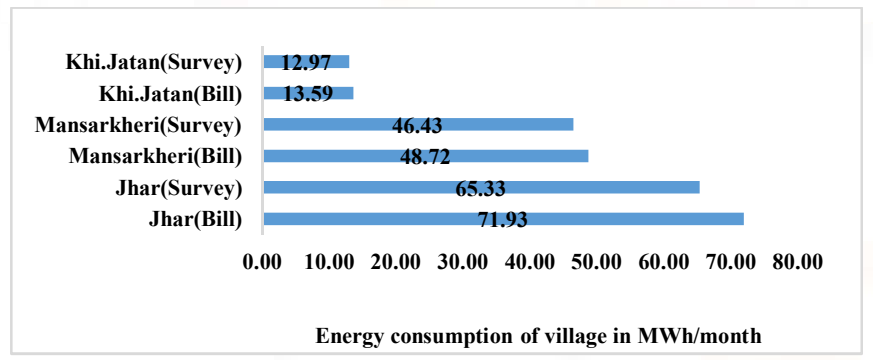

Figure-4: Energy consumption of village in MWh/month

The plots between survey data and bill data have also been plotted for each energy consumption parameters and shown in figures-1 to figure-7.

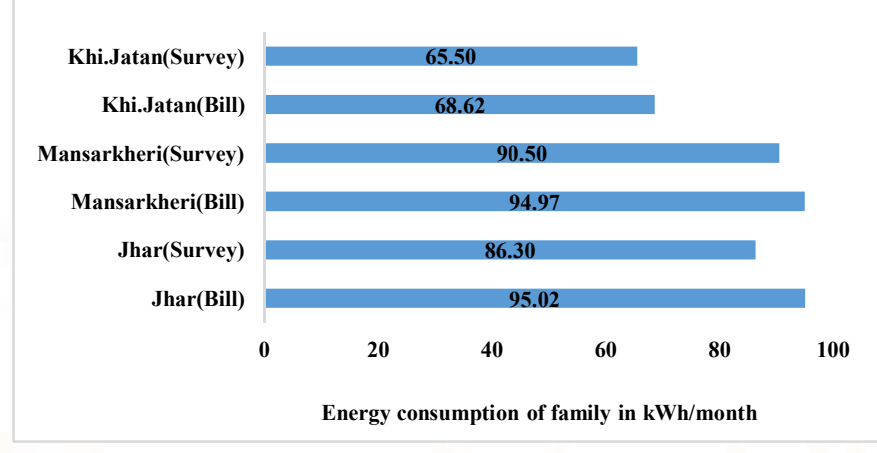

Figure -5 Energy consumption of family in $\mathrm{kWh} / \mathrm{month}$

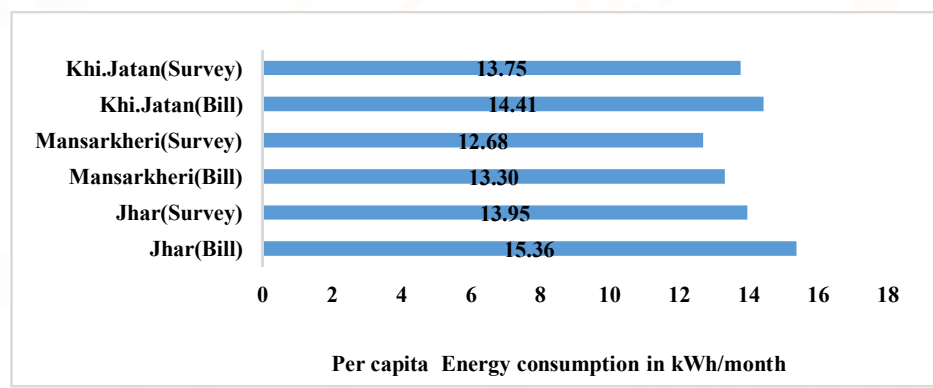

Figure-6: Per capita energy consumption in $\mathrm{kWh} / \mathrm{month}$

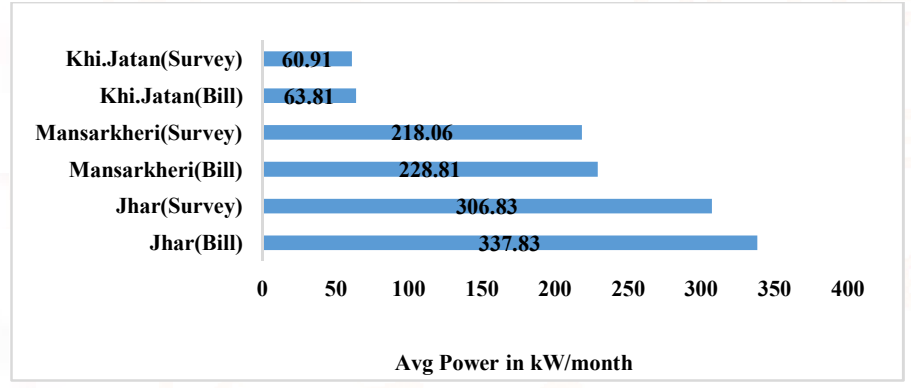

Figure-7: Average power requirement in $\mathrm{kW} / \mathrm{month}$

\section{CONCLUSIONS}

In this study electricity consumption data is collected by survey as well as local electricity Distribution Company. On the basis of these data, energy consumption parameters i.e. A-Energy consumption of villages in MWh/annum, B-Energy consumption of family in $\mathrm{kWh} /$ annum, C-Per capita Energy consumption in $\mathrm{kWh} /$ annum, D-Energy consumption of villages in $\mathrm{MWh} /$ month, E- Energy consumption of family in $\mathrm{kWh} / \mathrm{month}$, F- Per capita Energy consumption in $\mathrm{kWh} / \mathrm{month}$, G-Average Power in $\mathrm{kW} /$ month, have been calculated. Graphical representations also have been plotted between survey data and bill data for the purpose of comparative view. By viewing the graphical plots of energy consumption parameters it can be concluded that 
Energy consumption parameters A, B, C, D, E, F, G calculated by survey data are found to be less for each village as compared to energy consumption parameters calculated by bill data. Bill data therefore should be taken as reference data for designing any electrical power generation facility for the villages, instead of survey data because bill data are based on the meter readings of the individual households which shows the actual electricity consumed by the household.

\section{REFERENCES}

[1] R.D.Prasad, R.C.Bansal and A.Raturi, Multifaceted energy planning: A review, Renewable and Sustainable Energy Reviews 38 (2014) 686-699.

[2] T.Urmee, D.Harries and A.Schlapfer, Issues related to rural electrification using renewable energy in developing countries of Asia and Pacific, Renewable Energy 34 (2009) 354-357.

[3] N.Kautto and P.Peck, Regional biomass planningHelping to realise national renewable energy goals, Renewable Energy 46 (2012) 23-30.

[4] J.Singh, Overview of electric power potential of surplus agricultural biomass from economic, social, environmental and technical perspective- $\mathrm{A}$ case study of Punjab, Renewable and Sustainable Energy Reviews 42 (2015) 286-297.

[5] A.K.Bhandari and C.Jana, A comparative evaluation of household preferences for solar photovoltaic standalone and mini-grid system: An empirical study in a coastal village of Indian Sundarban, Renewable Energy 35 (2010) 2835-2838.

[6] I.Purohit and P.Purohit, Techno-economic evaluation of concentrating solar power generation in India, Energy Policy 38 (2010) 3015-3029

[7] Population census data of villages Jhar, Mansarkheri and Khijooriya Jatan, website, http://censusindia.gov.in/, accessed on March 2015.

\section{BIOGRAPHIES}



Vikrram Singh Meena

M.Tech- Renewable Energy

Malaviya National Institute Of

Technology, Jaipur, India

B.Tech- Electrical Engineering

Jamia Millia Islamia, New Delhi, India

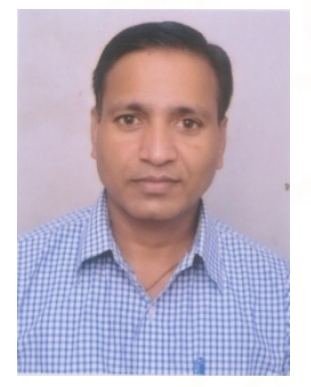

Dr. G. D. Agrawal

Associate Professor

Dept. of Mechanical Engineering, Malaviya National Institute Of Technology, Jaipur, India Ph.D.-Energy Recovery From MSW M.Tech.-Thermal Engineering B.E.-Mechanical Engineering 